\title{
The Characteristic of Spatial Form of Traditional Tibetan Settlement in Aba County of Sichuan Pro. China Based on the Perspective of Typological
}

\author{
Zaichang Zhou, Xuming Yang, Na Li \\ Department of Urban Planning and Architecture, Southwest University for Nationalities, Chengdu, China
}

Email address:

miragechou@126.com (Zaichang Zhou),695799399@qq.com (Xuming Yang),854175960@qq.com (Na Li)

To cite this article:

Zaichang Zhou, Xuming Yang, Na Li. The Characteristic of Spatial Form of Traditional Tibetan Settlement in Aba County of Sichuan Pro. China Based on the Perspective of Typological. International Journal of Environmental Protection and Policy. Vol. 4, No. 6, 2016 , pp. 155-161. doi: $10.11648 /$ j.ijepp.20160406.11

Received: October 29, 2016; Accepted: November 28, 2016; Published: December 1, 2016

\begin{abstract}
This paper takes the integral spatial form of traditional Tibetan settlement in Aba County Sichuan Pro. China as research object to process typology analysis. Based on the interactive analysis of natural environment and cultural background, this paper will discuss the typological characteristic of spatial form of traditional Tibetan settlement in Aba County.
\end{abstract}

Keywords: Typology, Tibetan Dwellings, Type of Settlement, Regionalism, Architecture

\section{Introduction}

Aba County locates in the northwest of western Sichuan plateau, which belongs to Aba Tibetan and Qiang Autonomous Prefecture. It is in the boundary of Sichuan, Gansu and Qinghai. It is one of the important Tibetan inhabited areas in China. In history, it had been the "national migration channel from northwest to southwest [1]". The landscape of the county is complicated: north and east is the hilly and flat plateau region based on plateau shallow hilly grassland. The west and middle is the basin and plateau mountainous region based on deep hilly. The south is mountain valley forest. The climate of county belongs to cold temperate semi humid monsoon climate in the plateau. Spring and fall are connected together and the rainy season is clear. It has sufficient sunshine and there is large temperature difference between day and night. Such geographical features make the character of local settlements and vernacular dwellings. The types are abundant. Colorful, shape and space combination are changeable. Harmonious unification of landform and landform characteristics shows the complexity and nationality of local architectural culture.

What the paper focuses is the complexity and diversity of Aba county of Tibetan settlements under the condition of changeable landform condition. It means that what kind of building adapts the change of landscape. Because Aba County has wide landscape and multiple settlements, the paper will make the settlement of different landscape mentioned above as the study object. It makes use of typology method to analyze the site selection, layout, road network, block land and building characteristics of settlement to explore the common regulation of settlement in different landscape condition.

\section{Description of Aba Tibetan County Location Factors}

Generally speaking, the location of Tibetan settlements in Aba County is affected from economy, natural environment and culture. Economic factor is production way and the natural environment factor includes water, topography, climate and elevation while the culture element is religion belief and national tradition.

\subsection{Production Method}

Production method is important reason to decide Tibetan village site selection. Most of the residents in north and east part of Aba County work on production in semi agriculture and graziery. The settlement patterns are in the scattered situation; the residents in the south work on half grazing state and the scale of settlement is based on the size of farmland. Most of building locates in bad farming land to save the farming land. Compared 
with other regions, the middle basin area has higher level of urbanization. The building quantity and density are higher too.

\subsection{Topography}

Water is the necessary basic protection of living and production. The first township level settlements in Aba County are built near rivers. The nearby is gentle zone with sunny exposure, which are the ideal settlement locations. Hence, most of the Tibetan settlements in Aba County are linear distribution, especially the southern mountain valley forest. The characters will be explained latter. In the strategy to fight for the high lighting efficiency, there will be different in various landscapes. In the valley of the East and West, most of the settlements are located in the gentle slope on the north side of the river along the contour, such as Shenzuo Village, Duowagongba Village (as shown in Figure 1 and Figure 2); in the valley of the South and North, most of the settlements are located on both sides of the valley and perpendicular to the direction of contour lines, such as Chali Village (as shown in Figure 3).

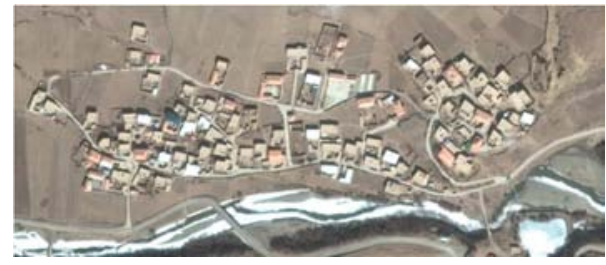

Figure 1. Shenzuo Village (satellite maps in this article are taken from Google Earth).

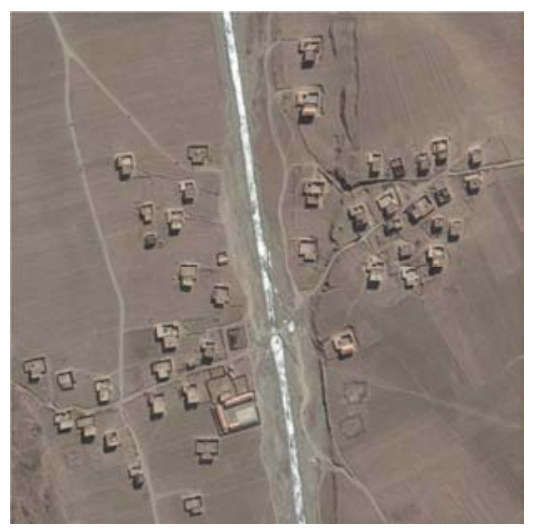

Figure 2. Donggou Village.

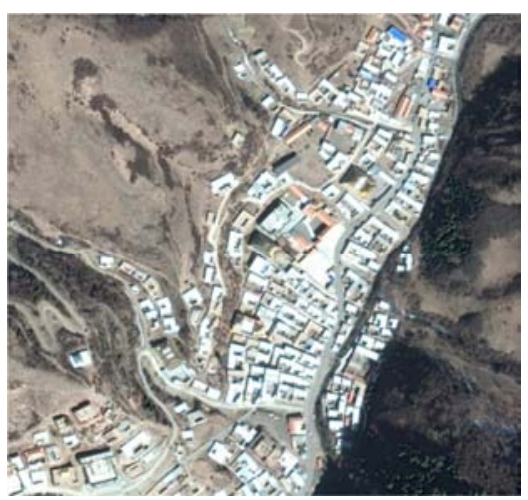

Figure 3. Part of Chali Village.

\subsection{Religion Belief}

Religion belief is one of the main ideologies in the Tibetan and it is the important component in the living of the people. There are so many Tibetan Buddhism sects in the county. There are settlements near holy mountains, holy lakes and temples with prosperous incense that are worshiped by the people, such as Gemogongba which is adjacent to the Gemo Temple. Temple and Lama Tower are the sprite activity centers of the village and they are also public activity centers. Many important ceremonies are held here, so temple and Lama Tower have special position in Tibetan village. In a word, the religion belief is one of the influences to the location.

\section{Spatial Form of Tibetan Settlement in Aba County}

\subsection{Hummocky and Flat Plateau Area}

\subsubsection{Site Selection_-Along the Gently River Bank}

Hummocky and flat plateau area mostly spreads over the north and east of Aba County, whose landform is flat and rich in water resource, making it an ideal place for settlement construction. Such environment can not only ensure that each household can possess relatively ample space for expansion, so as to meet the requirement of family development, but also guarantee the family daily production and living water demand. Under the construction of living spaces, lighting will also be taken into consideration.

Besides, during the construction process, in view of defensive property, some settlements will be built on the tableland a bit far from one side of the water source. Therefore, those settlements appear quite close, such as Kaxi Village in the northwest of Aba County. Likewise, for the sake of maximum use of water resource, some settlements are located on both sides of the river, which are relatively open, such as Donggou Village in the north.

\subsubsection{Form of the Settlements_-Scattering}

Flat plateau terrain provides enough space for settlement construction. Based on the lighting requirement, the space between each household is quite large and no distinct street or lane is formed, leading to the scattering settlement pattern. The layout of those settlements is usually loose and sparse, with large space between each household. Complicated road system becomes a bond connecting each building: Roads run around the stockaded village and several branch roads are derived to link each household; although buildings in the village are far away from each other, every household occupies certain space around the house.

Moreover, such terrain also offers sufficient space for settlement expansion. Road network form also varies according to the settlement site selection strategies. The main part of the road network in Kaxi Village is composed of one large and three small ring roads, from which other roads 
leading to each household are derived. The overall settlement is based on the tableland in the north of Provincial Rd S302. In the south, two major entrances of Kaxi Village are both located on the provincial road. Hence, the overall settlement presents a relatively close pattern (as shown in Figure 4). The road network of Donggou Village is mainly composed of three mutually connected roads (as shown in Figure 5). There are entrances in the north, south and east of the settlement. Therefore, compared with Kaxi Village, the Donggou Village is more open. Such sparse and scattering layout mode makes it hard to identify the border between clusters, which, further on, makes the border between settlements also indistinct.

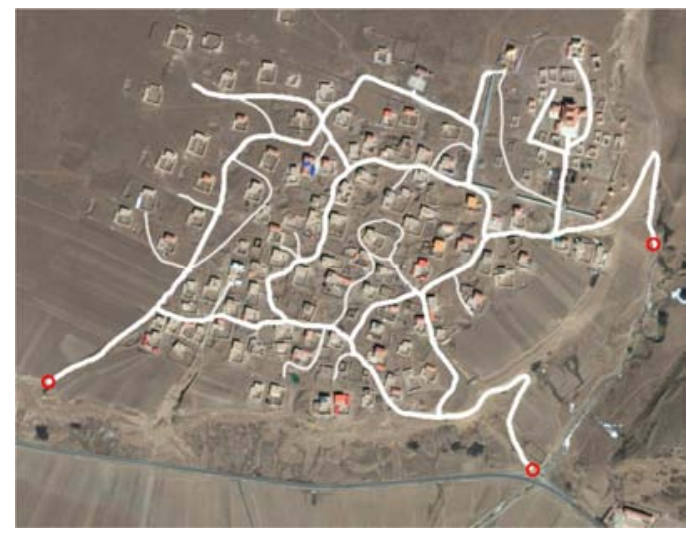

Figure 4. Entrances and Road Grid of Kaxi Village.

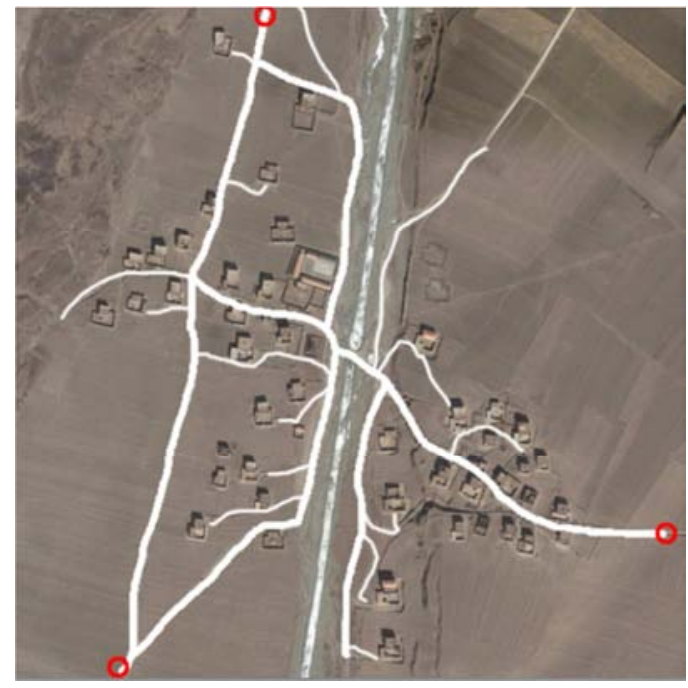

Figure 5. Entrances and Road Grid of Donggou Village.

\subsection{Plateau Basin and Plateau Mountain Areas}

\subsubsection{Site Selection_Close to the River Bank in the Basin}

The geographic location of the basin area along Provincial Rd. S302 in the middle of Aba County is quite superior: By virtue of affluent water resources, flat terrain and convenient traffic, many settlements with relative large scale are formed there. Restricted by the terrain, inside those dense settlements, relatively closely linked clusters are created (as shown in Figure 6). Compared with loose scattering layout, the construction space for each household in these settlements is quite small and the area of courtyard is compressed. In this case, lighting cannot be always taken into account.

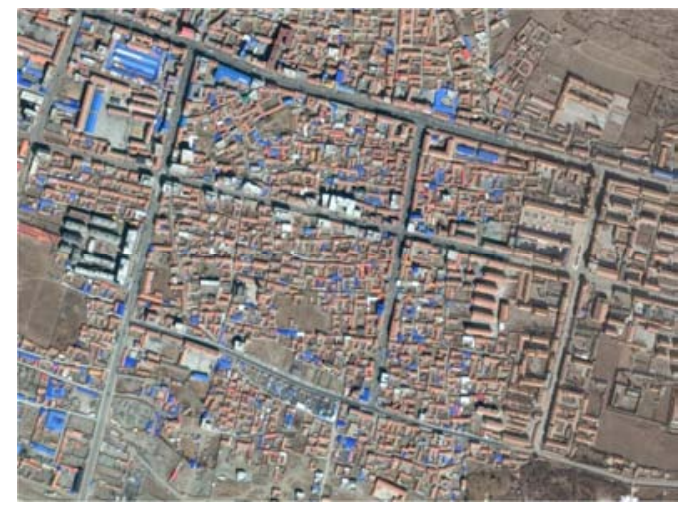

Figure 6. Closely Linked Clusters in Aba Town.

\subsubsection{Form of the Settlements-Network Type}

Such settlement form is also influenced by river, terrain and traffic routes. Outward main roads create linear village and town road networks, while mountains and rivers define the borders of settlements. Highly dense clusters form distinct streets and alleys.

Provincial Rd. S302 runs through the overall Aba Town from east to west. In the north of Aba Town there lies an important temple for Gelugpa: Kirti Monastery, which occupies a large majority of the area in the north of the road. The long and flat zone between the south and the river is partitioned by roads parallel to or perpendicular to Rd. S302 into regular grid layout clusters (as shown in Figure 6). Compared with other regions in Aba County, these clusters are densely-populated. In each cluster, there are dozens of hundreds of households. The cluster inside is partitioned and connected by various small roads (as shown in Figure 7). Mai'erma Township is located in the east of Aba Town, whose settlement pattern is similar to that of Aba Town. Provincial Rd. S302 divided Mai'erma Township from southeast to northwest into two parts. Clusters in the north centers on a south-north main road, spreading out grid-shaped road network in the latitudinal direction. For the north part, several long and narrow grid road networks are formed along with Provincial Rd. S302 (as shown in Figure 8).

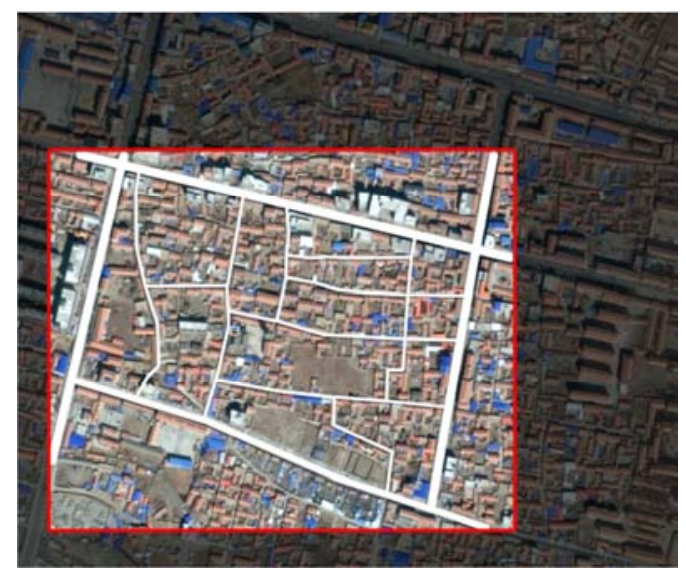

Figure 7. Part of Road Grid of Aba Town. 


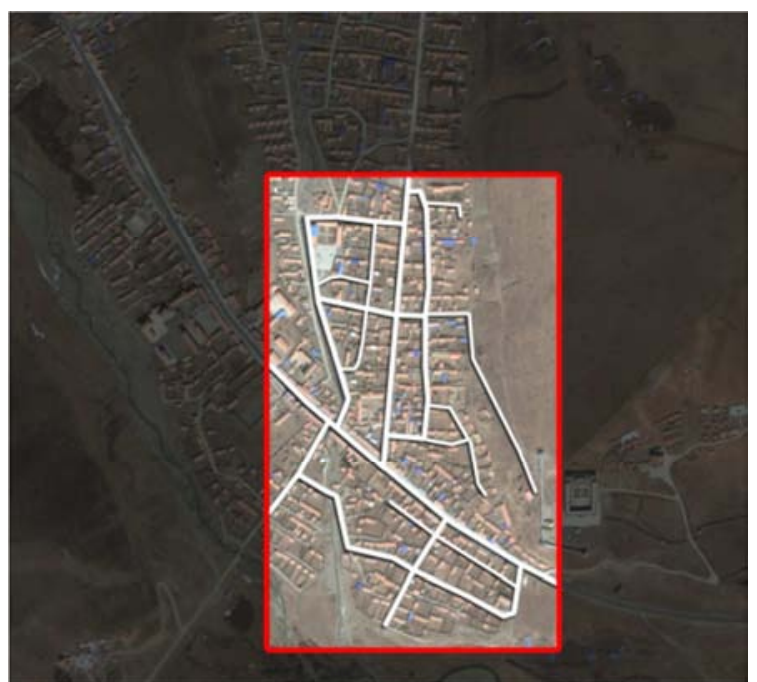

Figure 8. Part of Road Grid of Mai'erma Village.

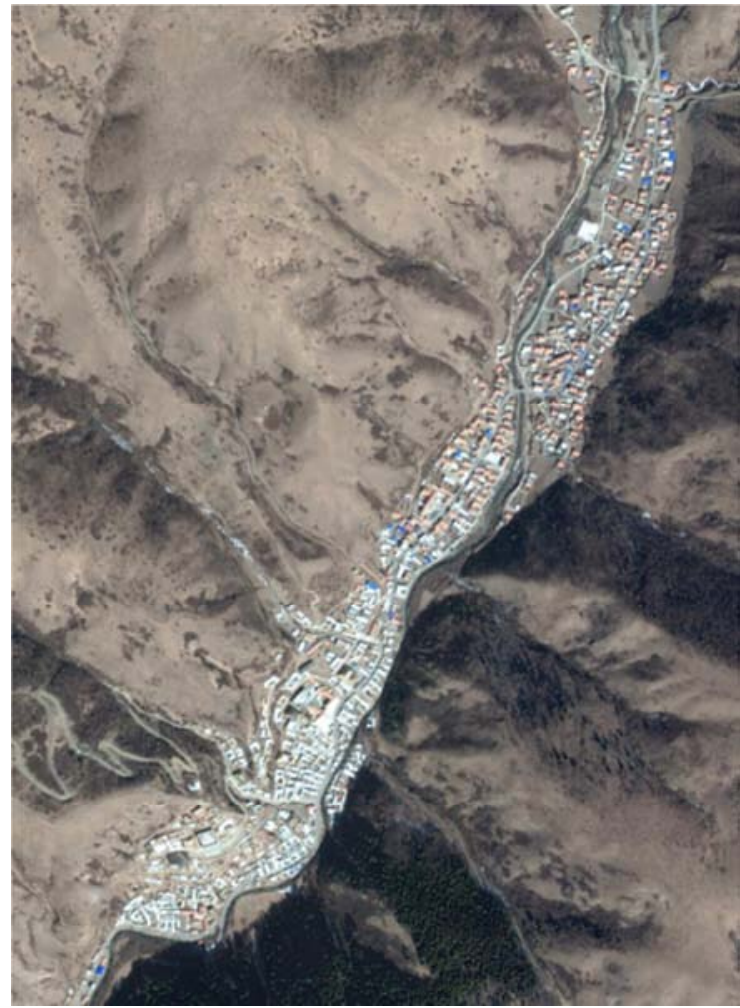

Figure 9. Chali Village.

\subsection{Alpine and Gorge Forest Region}

\subsubsection{Site Selection—Gently Areas Along the River Bank in Gorge}

Settlements in alpine and gorge forest region are greatly restricted by terrain compared with aforementioned two terrains. Therefore, the strategic core of the formation of settlements is full use of land on the premise of saving land. Those settlements are usually located at flat zones in the river valley along river or roads (as shown in Figure 9). Roads, rivers and mountains become natural boundaries of clusters. The formation pattern of each cluster varies with the changes of terrains. Those clusters not only meet the lighting requirement to the greatest extent, but also bring forth pleasant landscape to residents (as shown in Figure 10).

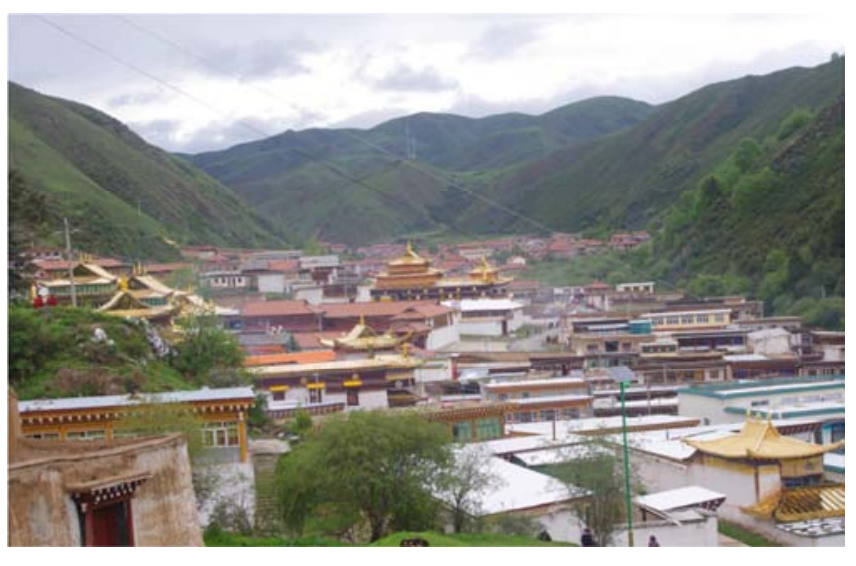

Figure 10. Chali Village.

\subsubsection{Form of the Settlements_-Organic Linear Layout}

As stated earlier, such type of settlements is often organic constitution of one or more clusters in the flat zone along the rivers. These clusters extend along roads or rivers in the valley, naturally forming linear layout form and presenting certain extensibility. Compared with other layout patterns, linear layout is dynamic; the principal axis along the river or road becomes a public activity center for cluster. Buildings and roads are directly connected. Hence, roads are not only places of interaction activities between villages and undertake the responsibilities of transportation and connection, but also become villagers' commodity exchange places. Such linear layout intensifies spatial connections and enhances the sense of depth, which has clear lines as well as strong structural property and orderliness. Individual building is greatly different from scattering building, which does not present the sense of a certain territory, but in general, shows the dynamics of village and enhances the flow of axis; when the single line growth of village exceeds a certain range, branches that cross main roads in the village will appear and construction area will be extended and radiated to hillside, generating T-shaped, cross-shaped and inverse V-shaped road networks. There comes composite space.

"Organic" is reflected in the different strategies of various groups constitution, as shown in Figure 11, the road network of two adjacent group in Chali Town has a great difference: the northeast one has a ring road as the core, which links surrounding households with radiation branches; and the southwest one has a main road as the core with fish bone shaped distribution. But its ultimate goal is to maximize the use of terrain, save arable land, expand the daylight area. As shown in Figure 12- Figure 13: Shenzuo village is consisted of a number of small groups along the north shore, the two main road roughly consistent with the contour line. Generally speaking, in the valleys of the north and south, houses are usually arranged along the vertical direction of the contour lines; in the East and west valley areas, houses are arranged along the contour lines. 


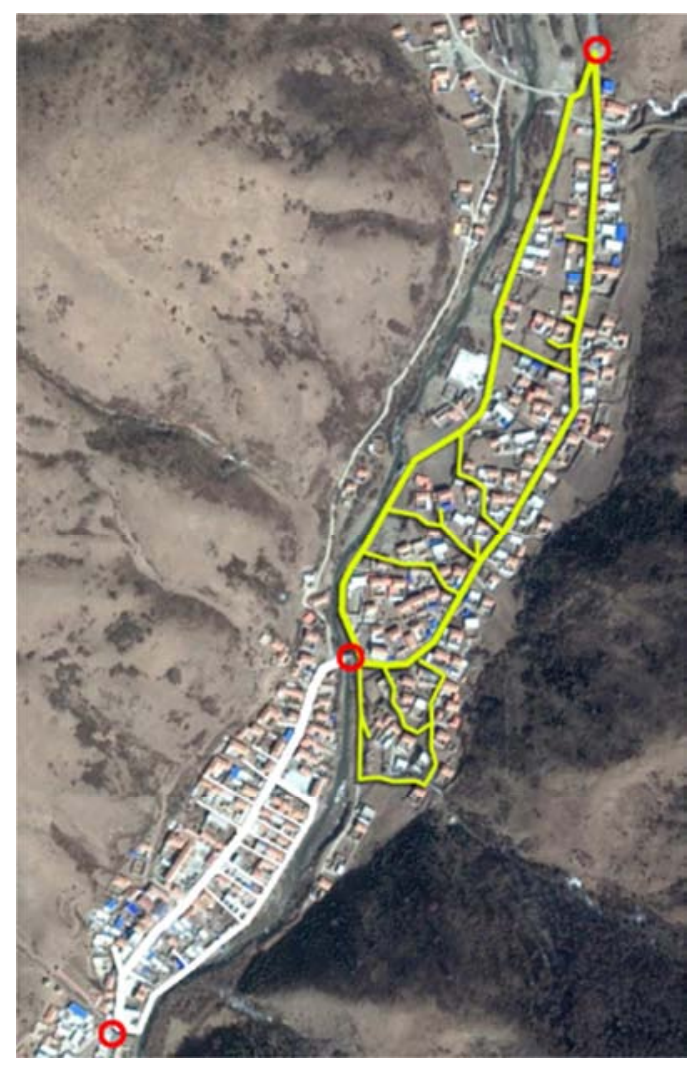

Figure 11. Part of Entrance and Road Grid of Chali Village.

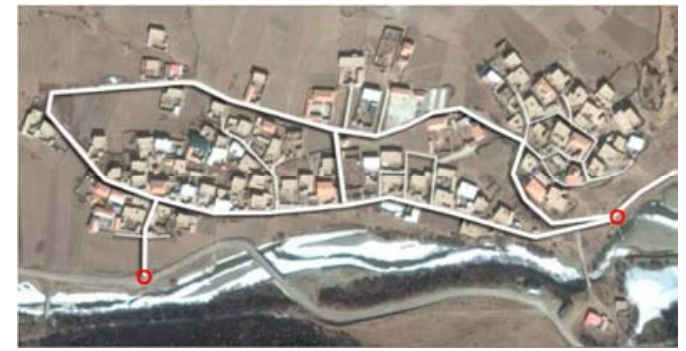

Figure 12. Entrance and Road Grid of Shenzuo Village.

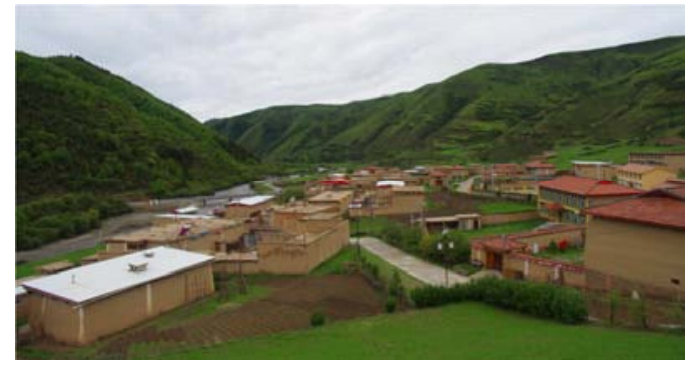

Figure 13. Shenzuo Village.

\section{The Characteristic of Spatial Form of Traditional Tibetan Settlement in Aba County}

According to the analysis above, it can be seen that the shape of the settlement is mainly affected by the combination of topography and form. Therefore, take the forms of groups as the basis, combined with the terrain of the settlement, the
Tibetan settlements in Aba County can be divided into three types: scattered type, network type and line type, and according to the terrain where those settlements are located in, they can be further divided into six subtypes (as shown in Figure 14).

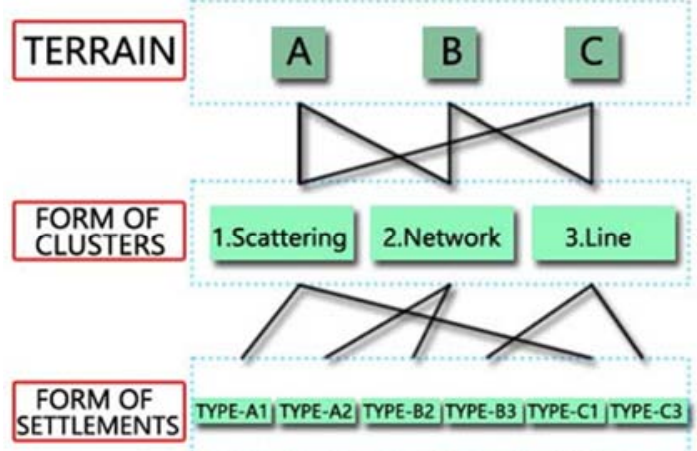

Figure 14. The Relationship between Terrain, Form of Clusters and Form of Settlements.

TYPE-A1: Scattering type settlements in hummocky and flat plateau areas. Typical settlement: Andou Village (as shown in Figure 15)

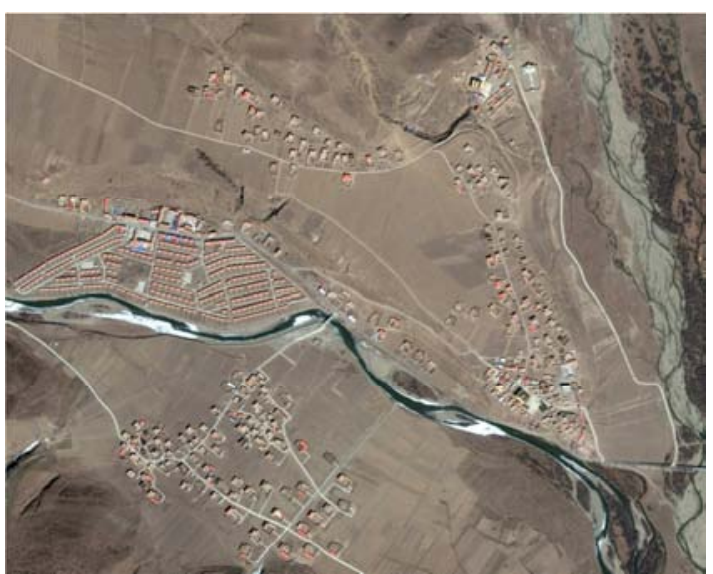

Figure 15. TYPE-A1 Typical Settlement: Andou Village.

TYPE-C1: Scattering type settlements in alpine and gorge forest region. Typical settlement: Kuasha Village (as shown in Figure 16).

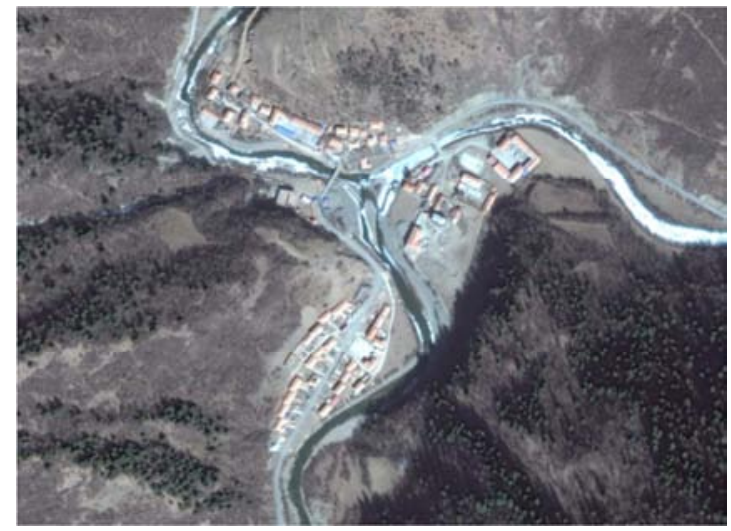

Figure 16. TYPE-C1 Typical Settlement: Kuasha Village. 
TYPE-A2: Network type settlement in hummocky and flat plateau areas. Typical settlement: Jialuo Village.

TYPE-B2: Network type settlement in plateau basin and plateau mountain areas. Typical settlement: Mai`erma Village.

TYPE-B3: plateau basin and plateau mountain areas. Typical settlement: Maikun Village (as shown in Figure 17).

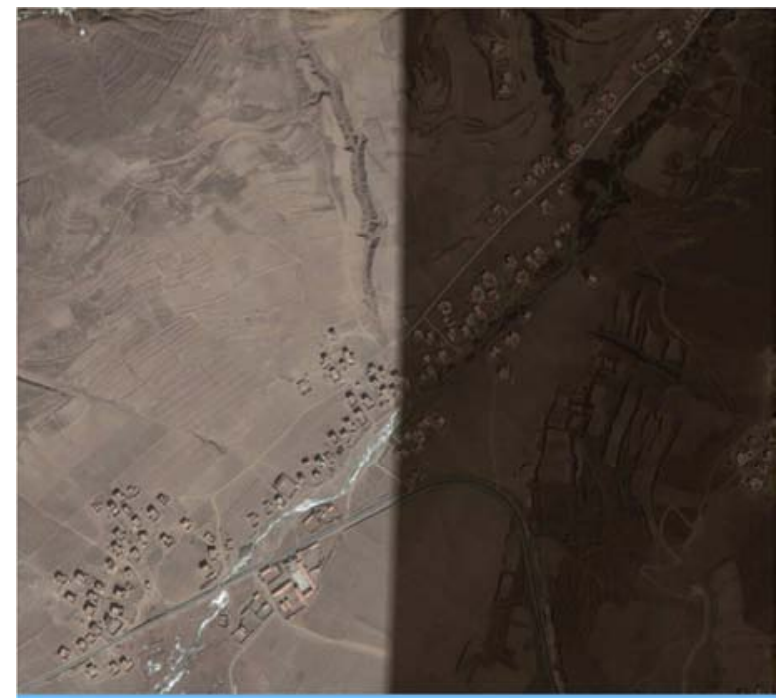

Figure 17. TYPEB3 Typical Settlement: Maikun Village.

TYPE-C3: line type settlements in alpine and gorge forest region. Typical settlement: Chali Village (as shown in Figure 18).

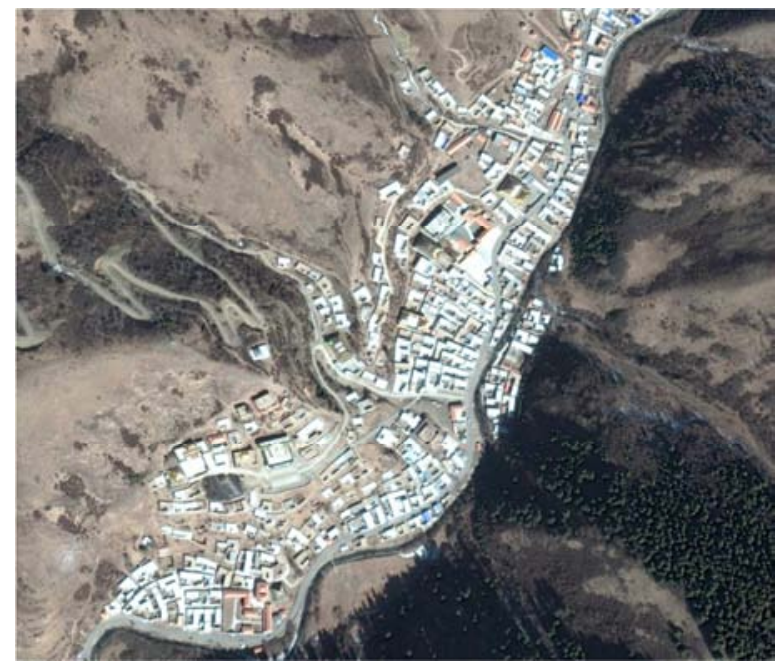

Figure 18. TYPEC3 Typical settlement: Chali Village(Part).

Scattering type settlements preform a great difference due to the locations: the form of Andou Village in hummocky and flat plateau is quite different from Kuasha Village's in alpine and gorge forest, but also the scale of clusters and the consist of buildings. The single building in the Andou Village is composed of the main building, the secondary building, the auxiliary space and the courtyard. The courtyard space in the households in alpine and gorge forest is highly reduced, and even some households have no enclosed courtyard. The same difference is also reflected in the line type settlements in plateau basin and plateau mountain areas and those in alpine and gorge forest areas (as shown in Figure 17-18). Compared to the settlements in hummocky and flat plateau areas and plateau basin and plateau mountain areas, the border of the settlements in alpine and gorge forest is determined by the terrain, and it is very easy to identify; while the borders of the settlements in the terrains mentioned former are relative indistinct.

Table 1. Types of all Villages and Towns in Aba County.

\begin{tabular}{ll}
\hline Andou Village & TYPE-A1 \\
Qiujima Village & TYPE-A1 \\
Jialuo Village & TYPE-A2 \\
Wa`erma Village & TYPE-B2 \\
Mai'erma Village & TYPE-B2 \\
Dege Village & TYPE-B2 \\
Aba Town & TYPE-B2 \\
Gemo Village & TYPE-B2 \\
Hezhi Village & TYPE-B3 \\
Siwa Village & TYPE-B3 \\
Maikun Village & TYPE-B3 \\
Longzang Village & TYPE-B3 \\
Jia`erduo Village & TYPE-B3 \\
Luo`erda Village & TYPE-C1 \\
Kuasha Village & TYPE-C1 \\
Rong`an Village & TYPE-C3 \\
Chali Village & TYPE-C3 \\
Anqiang Village & TYPE-C3 \\
Kehe Village & TYPE-C3 \\
\hline
\end{tabular}

The Tibetan settlements in Aba County, locate in the seemingly random accidental mountains (as shown in Figure 19), and show a great difference, but it contains the same architectural wisdom: making full use of terrain to create the most suitable living environment, and do not stick to stereotypes. The diversity of the type of settlement is the result of the continuous attempt to coordinate the relationship between the settlement and the terrain, water and sunlight. The inherent rule of which is worth deeply studying and thinking.

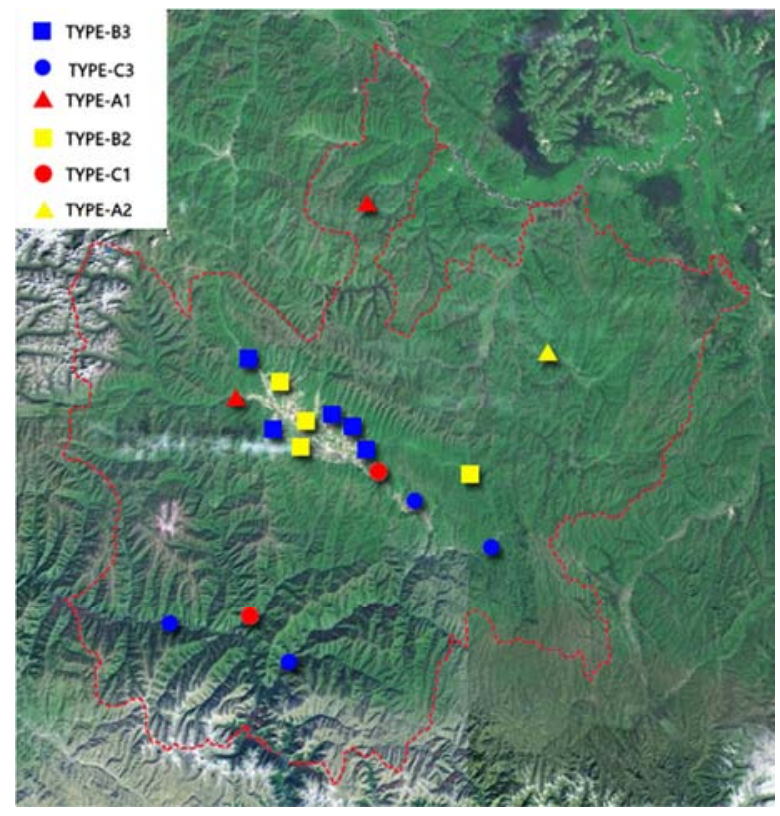

Figure 19. Types of all Villages and Towns in Aba County. 


\section{Conclusion}

Aba Tibetan settlement appears to be arranged in the mountain accidentally and randomly, in fact, the layout is composed organically in order to adapt to different geographical conditions. According to the type of settlement above, we can conclude: scattering type is a relatively loose and sparse layout; line type is influenced by the topography, the layout is relatively close; high population density caused by relative important location urge the network type to emerge. No matter loose and sparse or close, these forms reflect the traditional Tibetan construction method which utilizes limited natural resources organically in the Aba. This method has a strong guiding significance for the town planning and management of Tibetan settlements in the context of the new era.

Traditional Tibetan settlements and dwellings in Aba County as an important branch of Tibetan architectural culture, reflect the Tibetan people's experience and wisdom which is accumulated in the long-term utilizing of nature. It is a good example of harmonious coexistence of human and nature. Different types of settlements suit their measures to local conditions, reflecting the Neo-vernacular Architecture values, namely "born here, grow here". Keep studying of this subject further, extract the outstanding factors of traditional settlements and residential buildings, which will provide theoretical basis and practical guiding significance for updating and improving the traditional settlement and building new settlements.

\section{Acknowledgements}

This research is funded by Southwest University for Nationalities Graduate Innovative Research Project (CX2015SZ013), the Southwest University for Nationalities research project of the Sichuan regional adaptation of Tibetan dwellings on analysis of ecological prototype (2015NYB01).

\section{References}

[1] S. Shuo. Corridor of the Tibet-Yi Minority [M]. Chengdu: Sichuan People's Publishing House, 2009.

[2] Q. Zhao. Research on the Ecological Experiences and Pattern Language of Traditional residential buildings [D]. Xi'an: Xi'an University of Architecture and Technology, 2005.

[3] J. H. Li, "The Overall Space and Form Pattern of Jiarong Tibetan Traditional Settlement", Urbanism and Architecture, pp. 36-39, 2011.

[4] T. Zhang. Study on Climate Adaptability of Typical Traditional Dwellings Envelope [D]. Xi`an: Xi an University of Architecture and Technology, 2013.

[5] Q. S. Ye. Tibetan Residence in Sichuan [M]. Chengdu: Sichuan Minorities Press, 1989.

[6] J. Xie. Study on the Traditional Tibetan Residential of Ganzi areas in southeastern Sichuan. Xi'an: Xi'an University of Architecture and Technology, 2010.

[7] Q. Zhao, W. Zhou, J. P. Liu, "Ecological Building Experiences in Chinese Traditional Dwellings" New Architecture,pp.8-9, 2005. 4.

[8] L. L. He. Ecological Experience and Enlightenment of Traditional Dwellings in Sichuan Basin [D]. Guangzhou: South China University of Technology, 2014.

[9] Y. Wang. Research of update design of Jiarong Tibetan dwelling [D]. Xi'an: Xi'an University of Architecture and Technology, 2014.

[10] G. Mao, "Ecological View: the Architecture and Habitation in the High Southwest Mountain Region" [M], Nanjing: Southeast university press, 2003.

[11] L. Zhao. Study on the Traditional Tibetan Residential construction mode of Jia Rong in west Sichuan [D]. Xi an: Xi an University of Architecture and Technology, 2014. 